\title{
Existence of Solutions to Elliptic Problem with Convection Term and Lower-Order Term
}

\author{
Xiaohua He, ${ }^{1}$ Shuibo Huang $\mathbb{D}^{1,2}$ Qiaoyu Tian, ${ }^{1}$ and Yonglin $X u^{1}$ \\ ${ }^{1}$ School of Mathematics and Computer Science, Northwest Minzu University, Lanzhou, Gansu 730030, China \\ ${ }^{2}$ Key Laboratory of Streaming Data Computing Technologies and Application, Northwest Minzu University, Lanzhou, \\ Gansu 730030, China
}

Correspondence should be addressed to Shuibo Huang; huangshuibo2008@163.com

Received 18 September 2020; Accepted 12 October 2020; Published 28 October 2020

Academic Editor: J. Vanterler da C. Sousa

Copyright (c) 2020 Xiaohua He et al. This is an open access article distributed under the Creative Commons Attribution License, which permits unrestricted use, distribution, and reproduction in any medium, provided the original work is properly cited. In this paper, we establish the existence of solutions to the following noncoercivity Dirichlet problem
$\begin{cases}-\operatorname{div}(M(x) \nabla u)+|u|^{p-1} u=-\operatorname{div}(u E(x))+f(x), & x \in \Omega, \\ u(x)=0, & x \in \partial \Omega,\end{cases}$
belongs to the Lebesgue space $L^{m}(\Omega)$ with $m \geq 1, p>0$. The main innovation point of this paper is the combined effects of the convection terms and lower-order terms in elliptic equations.

\section{Introduction}

The main purpose of this paper is to prove the existence of solutions to the following elliptic boundary value problem

$$
\begin{cases}-\operatorname{div}(M(x) \nabla u)+|u|^{p-1} u=-\operatorname{div}(u E(x))+f(x), & x \in \Omega, \\ u(x)=0, & x \in \partial \Omega,\end{cases}
$$

where $\Omega$ is a bounded smooth subset of $\mathbb{R}^{N}(N>2)$ with $0 \in \Omega$, and $M: \Omega \longrightarrow \mathbb{R}^{N^{2}}$ is a bounded measurable matrix, which satisfies the following conditions: there are two positive constants $\alpha$ and $\beta$, such that, for a.e. $x \in \Omega$ and every $\xi \in \mathbb{R}^{N}$,

$$
\alpha|\xi|^{2} \leq M(x) \xi \cdot \xi, \quad|M(x)| \leq \beta
$$

Moreover, $E(x)$ is a measurable vector field, which satisfies

$$
|E| \leq \frac{A}{|x|}, A \in \mathbb{R}^{+},
$$

$f(x)$ is a measurable function which satisfies

$$
f \in L^{m}(\Omega)(m \geq 1)
$$

where $L^{m}(\Omega)$ represents the Lebesgue space.

When the problem (1) without the lower-order terms, it becomes the following elliptic Dirichlet problem

$$
\begin{cases}-\operatorname{div}(M(x) \nabla u)=-\operatorname{div}(u E(x))+f(x), & x \in \Omega, \\ u(x)=0, & x \in \partial \Omega,\end{cases}
$$


Problem (5) was studied by Boccardo in a series of papers. More precisely, in [1], the existence of solutions $u$ to problem (5) was proved provided $E \in\left(L^{N}(\Omega)\right)^{N}$ :

(i) If $f \in L^{m}(\Omega)$ with $2 N / N+2 \leq m<N / 2$, then $u \in$ $W_{0}^{1,2}(\Omega) \cap L^{m^{* *}}(\Omega)$, where $m^{* *}=N m / N-2 m$

(ii) If $f \in L^{m}(\Omega)$ with $1<m<2 N / N+2$, then $u \in$ $W_{0}^{1, m^{*}}(\Omega)$, where $m^{*}=N m / N-m$

(iii) If $f \in L^{m}(\Omega)$ with $m>N / 2$, then $u \in W_{0}^{1,2}(\Omega) \cap$ $L^{\infty}(\Omega)$

(iv) If $f \in L^{1}(\Omega)$, then $u \in W_{0}^{1, q}(\Omega)$, where $q<N / N-1$

When $f \in L^{m}(\Omega)$ and vector field $E$ satisfies (3), which does not belong to $\left(L^{N}(\Omega)\right)^{N}$, Boccardo [2] shown that

(i) If $A<\alpha(N-2 m) / m$ and $2 N / N+2 \leq m<N / 2$, then $u \in W_{0}^{1,2}(\Omega) \cap L^{m^{* *}}(\Omega)$

(ii) If $A<\alpha(N-2 m) / m$ and $1<m<2 N / N+2$, then $u$ $\in W_{0}^{1, m^{*}}(\Omega)$

(iii) If $A<\alpha(N-2)$ and $m=1$, then $\nabla u \in\left(M^{N / N-1}(\Omega)\right)^{N}$ and $u \in W_{0}^{1, q}(\Omega)$, where $q<N / N-1$

Furthermore, in the same paper, the existence of entropy solutions to problem (5) also be considered provided $E \epsilon$ $\left(L^{2}(\Omega)\right)^{N}$ and $f \in L^{1}(\Omega)$.

Recently, continuation of [2], Boccardo and Orsina [3] studied the existence of distributional solution $u \in W_{0}^{1, q}(\Omega)$ to problem (5) with $q<N \alpha / A+\alpha$ provided $\alpha(N-2) \leq A<$ $\alpha(N-1)$ and $f \in L^{1}(\Omega)$. Moreover, $u$ verifies a prior estimation:

$$
\left(\int_{\Omega}|\nabla u|^{q}\right)^{1 / q} \leq C_{E}\|f\|_{L^{1}(\Omega)} .
$$

The constant $C_{E}$ lies on $E, \alpha$, and $\Omega$. Some other results about noncoercivity elliptic problems see [4-16] and reference therein.

It is well known that the presence of lower-order term will improve the regularity properties of the solutions. When $p=1$ and $M(x)$ satisfying (2), Boccardo [2] shown that the existence of entropy solution $u$ of (1) provided $E \in$ $\left(L^{2}(\Omega)\right)^{N}$ and $f \in L^{1}(\Omega)$.

Moreover, $u$ verifies the following estimations:

$$
\begin{gathered}
\int_{\Omega}|u| \leq \int_{\Omega}|f|, \\
\int_{\Omega}|\nabla \log (1+|u|)|^{2} \leq \frac{1}{\alpha^{2}} \int_{\Omega}|E|^{2}+\frac{2}{\alpha} \int_{\Omega}|f|, \\
\frac{\alpha}{2} \int_{\Omega}\left|\nabla T_{k}(u)\right|^{2} \leq \frac{k^{2}}{2 \alpha} \int_{\Omega}|E|^{2}+k \int_{\Omega}|f| .
\end{gathered}
$$

Furthermore, there is a weak solution $u \in W_{0}^{1.2}(\Omega) \cap$ $L^{p+1}(\Omega)$ of (1) provided (2) holds, $p>N+2 / N-2, f \in$ $L^{p+1 / p}(\Omega)$, and $E \in\left(L^{2(p+1) / p-1}(\Omega)\right)^{N}$.

For some other results about elliptic problems with lower-order terms, see [17-26] and reference therein.

With motivation from the results of the above-cited papers, the main goal of this paper is to further study the regularity of solutions to problem (1) with $f \in L^{m}(\Omega)(m \geq 1)$. The main features of this paper are the presence of the convection term $\operatorname{div}(u E(x))$, which leads to the noncoercivity of $-\operatorname{div}(M(x) \nabla u)+\operatorname{div}(u E(x))$ in $W_{0}^{1,2}(\Omega)$. Therefore, in order to overcome the coercivity difficulty, we use truncation technique and consider the corresponding approximate Dirichlet problem, see (19) for more details.

The main results are the following:

Theorem 1. Suppose that $\Omega$ is a bounded smooth domain of $\mathbb{R}^{N}(N>2)$ with $0 \in \Omega$ and (2)-(4) hold.

(a) There is a weak solution $u \in W_{0}^{1.2}(\Omega) \cap L^{p+1}(\Omega)$ to problem (1) provided $A<\alpha(N-2) / 2$ and $f \in L^{m}(\Omega)$ with $1 \leq m \leq 1+(1 / p)$

(b) There is a weak solution $u \in L^{m_{p}}(\Omega)$ to problem (1) provided $A<\alpha(N-2) / p(m-1)+1$ and $f \in L^{m}(\Omega)$ with $m>p+1 / p$. Furthermore,

$$
\|u\|_{L^{m_{p}(\Omega)}} \leq C\|f\|_{L^{m}(\Omega)},
$$

where

$$
m_{p}=\frac{(p(m-1)+1) N}{N-2} .
$$

Remark 2. A point worth emphasizing is that our results further refine the conclusions of [2]. More precisely, under different assumptions on $E$, we give the existence of solutions to problem (1) with $f \in L^{m}(\Omega)$ for $1 \leq m \leq p+1 / p$ and $m>$ $p+1 / p$, respectively, rather than $f \in L^{p+1 / p}(\Omega)$.

Remark 3. Obviously,

$$
m_{p}=\frac{(p(m-1)+1) N}{N-2} \longrightarrow+\infty \text { as } p \longrightarrow+\infty
$$

which shows the regularizing effect of the lower-order term for the regularity properties of the solutions to problem (1).

Remark 4. It is clear that

$$
A<\frac{\alpha(N-2)}{p(m-1)+1} \longrightarrow 0 \text { as } p \longrightarrow+\infty
$$

which shows that the lower-order term intensifies the requirement on $E$.

The paper is organized as follows. In Section 2, we give some definitions and lemmas. In Section 3, the Proof of Theorem 1 is given. 


\section{Useful Tools and Function Setting}

In order to prove Theorem 1, the following basic definitions and lemmas are needed. First of all, we give the definitions of weak solution to (1).

Definition 5. We say that $u \in W_{0}^{1,2}(\Omega)$ is a weak solution to problem (1), if $\int_{\Omega}|u|^{p-1} u d x<\infty$ and

$$
\int_{\Omega} M(x) \nabla u \nabla v+\int_{\Omega}|u|^{p-1} u v=\int_{\Omega} u E(x) \nabla v+\int_{\Omega} f v,
$$

for every $v \in W_{0}^{1,2}(\Omega)$.

The following is the definition of the truncation function.

Definition 6. For $\forall k \geq 0, s \in \mathbb{R}$, the truncation function defined by

$$
T_{k}(s)=\max \{-k, \min \{k, s\}\}, G_{k}(s)=s-T_{k}(s) .
$$

Now, let us briefly recall the Sobolev's embedding theorem.

Lemma 7. Assume that $p=2$, then there is a normal number $S$ , such that, for $\forall u \in C_{0}^{\infty}\left(\mathbb{R}^{N}\right)$ satisfies

$$
\|u\|_{2^{*}} \leq S\|\nabla u\|_{2^{\prime}}
$$

where

$$
2^{*}=\frac{2 N}{N-2} \text {. }
$$

The following Hölder inequality plays an important role in this paper.

Lemma 8 (Hölder inequality). Assume that $1<p, q<\infty$, $(1 / p)+(1 / q)=1$. Then, if $u \in L^{p}(\Omega), v \in L^{q}(\Omega)$, we have

$$
\int_{\Omega}|u v| d x \leq\|u\|_{L^{p}(\Omega)}\|v\|_{L^{q}(\Omega)} .
$$

The results of the Hardy inequality and its generalization can be founded in [27-29]. In this paper, we use the following Hardy inequality repeatedly.

Lemma 9 (see [3]). For $\forall v(x) \in W_{0}^{1,2}(\Omega)$, we have

$$
H\left(\int_{\Omega} \frac{|v|^{2}}{|x|^{2}}\right)^{1 / 2} \leq\left(\int_{\Omega}|\nabla v|^{2}\right)^{1 / 2}
$$

where

$$
H=\frac{N-2}{2} .
$$

\section{Proof of Main Theorem}

In this part, we are going to give the Proof of Theorem 1 in a similar way as $[2,17-21,23,26]$. In order to do this, first of all, we consider the following approximate problem:

$$
\begin{cases}-\operatorname{div}\left(M(x) \nabla u_{n}\right)+\left|u_{n}\right|^{p-1} u_{n}=-\operatorname{div}\left(\frac{u_{n}}{1+(1 / n)\left|u_{n}\right|} \frac{E(x)}{1+(1 / n)|E(x)|}\right)+f_{n}(x), & x \in \Omega, \\ u_{n}(x)=0, & x \in \partial \Omega,\end{cases}
$$

where

$$
f_{n}(x)=\frac{f(x)}{1+(1 / n)|f(x)|} .
$$

Let us start with the following conclusions.

First of all, the following lemma gives an information on the summability of $|u|^{p-1} u$.

Lemma 10. Let $f \in L^{m}(\Omega), m \geq 1$. Then, for every $n \in \mathbb{N}$, there exists a solution $u_{n} \in W_{0}^{1,2}(\Omega)$ to (19) such that

$$
\int_{\Omega}\left|u_{n}\right|^{p m} \leq \int_{\Omega}|f|^{m} .
$$

Proof. In order to get the estimates (21), we will consider the following two cases separately.

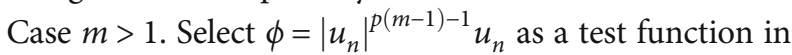
(19), by (3), we have

$$
\begin{aligned}
& \int_{\Omega} M(x) \nabla u_{n} \cdot \nabla\left|u_{n}\right|^{p(m-1)-1} u_{n}+\int_{\Omega}\left|u_{n}\right|^{p m} \\
& =\int_{\Omega} \frac{u_{n}}{1+(1 / n)\left|u_{n}\right|} \frac{E(x)}{1+(1 / n)|E(x)|} \cdot \nabla\left|u_{n}\right|^{p(m-1)-1} u_{n} \\
& \quad+\int_{\Omega} f_{n}(x)\left|u_{n}\right|^{p(m-1)-1} u_{n} \leq\left. A \int_{\Omega} \frac{u_{n}}{|x|}|\nabla| u_{n}\right|^{p(m-1)-1} u_{n} \mid \\
& \quad+\int_{\Omega} f_{n}(x)\left|u_{n}\right|^{p(m-1)-1} u_{n} .
\end{aligned}
$$


According to (2) and (22), we get

$$
\begin{aligned}
& \alpha p(m-1) \int_{\Omega}\left|\nabla u_{n}\right|^{2}\left|u_{n}\right|^{p(m-1)-1}+\int_{\Omega}\left|u_{n}\right|^{p m} \\
& \leq A p(m-1) \int_{\Omega} \frac{u_{n}}{|x|}\left|\nabla u_{n}\right|\left|u_{n}\right|^{p(m-1)-1}+\int_{\Omega} f_{n}(x)\left|u_{n}\right|^{p(m-1)-1} u_{n} \\
&=\left.\frac{2 A p(m-1)}{p(m-1)+1} \int_{\Omega} \frac{\left|u_{n}\right|^{p(m-1)+1 / 2}}{|x|}|\nabla| u_{n}\right|^{p(m-1)+1 / 2} \mid \\
& \quad+\int_{\Omega} f_{n}(x)\left|u_{n}\right|^{p(m-1)-1} u_{n} .
\end{aligned}
$$

Using the Hölder inequality and the Hardy inequality for the first term on the right of (23), we obtain

$$
\begin{aligned}
& \left.\frac{2 A p(m-1)}{p(m-1)+1} \int_{\Omega} \frac{\left|u_{n}\right|^{p(m-1)+1 / 2}}{|x|}|\nabla| u_{n}\right|^{p(m-1)+1 / 2} \mid \\
& \leq\left.\left.\frac{2 A p(m-1)}{[p(m-1)+1] H} \int_{\Omega}|\nabla| u_{n}\right|^{p(m-1)+1 / 2}\right|^{2} .
\end{aligned}
$$

Since

$$
\begin{aligned}
\alpha p & (m-1) \int_{\Omega}\left|\nabla u_{n}\right|^{2}\left|u_{n}\right|^{p(m-1)-1} \\
& =\left.\left.\frac{4 \alpha p(m-1)}{[p(m-1)+1]^{2}} \int_{\Omega}|\nabla| u_{n}\right|^{p(m-1)+1 / 2}\right|^{2} .
\end{aligned}
$$

Thus, taking into account (23)-(25), we arrive at

$$
\begin{aligned}
& {\left.\left.\left[\frac{4 \alpha p(m-1)}{[p(m-1)+1]^{2}}-\frac{2 A p(m-1)}{[p(m-1)+1] H}\right] \int_{\Omega}|\nabla| u_{n}\right|^{p(m-1)+1 / 2}\right|^{2}} \\
& \quad+\int_{\Omega}\left|u_{n}\right|^{p m} \leq \int_{\Omega} f_{n}(x)\left|u_{n}\right|^{p(m-1)-1} u_{n} .
\end{aligned}
$$

This fact leads to

$$
\int_{\Omega}\left|u_{n}\right|^{p m} \leq \int_{\Omega} f_{n}(x)\left|u_{n}\right|^{p(m-1)-1} u_{n}
$$

provided

$$
A<\frac{\alpha(N-2)}{p(m-1)+1}
$$

where $H=N-2 / 2$.

Applying the Hölder inequality on the right-hand side of (27), we get

$$
\int_{\Omega} f_{n}(x)\left|u_{n}\right|^{p(m-1)-1} u_{n} \leq\|f\|_{L^{m}(\Omega)}\left(\int_{\Omega}\left|u_{n}\right|^{p m}\right)^{1 / m^{\prime}},
$$

where $m^{\prime}=m / m-1$, which together with (27), implies that (21) holds.
When $m=1$, taking $T_{k}\left(u_{n}\right) / k$ as a test function in (19), according to (3), we have

$$
\begin{aligned}
\int_{\Omega} M(x) \nabla u_{n} \cdot \nabla \frac{T_{k}\left(u_{n}\right)}{k}+\int_{\Omega}\left|u_{n}\right|^{p-1} u_{n} \frac{T_{k}\left(u_{n}\right)}{k} \\
=\int_{\Omega} \frac{u_{n}}{1+(1 / n)\left|u_{n}\right|} \frac{E(x)}{1+(1 / n)|E(x)|} \\
\quad \cdot \nabla \frac{T_{k}\left(u_{n}\right)}{k}+\int_{\Omega} f_{n}(x) \frac{T_{k}\left(u_{n}\right)}{k} \\
\leq A \int_{\Omega} \frac{u_{n}}{|x|}\left|\nabla \frac{T_{k}\left(u_{n}\right)}{k}\right|+\int_{\Omega} f_{n}(x) \frac{T_{k}\left(u_{n}\right)}{k} .
\end{aligned}
$$

For the first term on the left-hand side of (30), we have

$$
\int_{\Omega} M(x) \nabla u_{n} \cdot \nabla \frac{T_{k}\left(u_{n}\right)}{k}=\frac{1}{k} \int_{\left|u_{n}\right| \leq k} M(x) \nabla u_{n} \cdot \nabla u_{n}
$$

For the first term on the right-hand side of (30), using the Hölder inequality and the Hardy inequality, we get

$$
A \int_{\Omega} \frac{u_{n}}{|x|}\left|\nabla \frac{T_{k}\left(u_{n}\right)}{k}\right|=\frac{A}{k} \int_{\left|u_{n}\right| \leq k} \frac{u_{n}}{|x|}\left|\nabla u_{n}\right| \leq \frac{A}{H k} \int_{\left|u_{n}\right| \leq k}\left|\nabla u_{n}\right|^{2} .
$$

Combining (30)-(31) with (2), we have

$$
\left(\frac{\alpha}{k}-\frac{A}{H k}\right) \int_{\left|u_{n}\right| \leq k}\left|\nabla u_{n}\right|^{2}+\int_{\Omega}\left|u_{n}\right|^{p} \frac{T_{k}\left(u_{n}\right)}{k} \leq \int_{\Omega} f_{n}(x) \frac{T_{k}\left(u_{n}\right)}{k} .
$$

Since $A<\alpha(N-2) / 2$, we obtain

$$
\int_{\left|u_{n}\right| \leq k}\left|u_{n}\right|^{p} \frac{T_{k}\left(u_{n}\right)}{k} \leq \int_{\left|u_{n}\right| \leq k} f_{n}(x) \frac{T_{k}\left(u_{n}\right)}{k} \leq \int_{\Omega} f_{n}(x) \leq \int_{\Omega}|f| .
$$
holds.

Fatou lemma implies, for $k \longrightarrow \infty$, the expression of (21)

Next, we will prove the following existence result.

Lemma 11. Assume (2), (3) hold with $A<\alpha(N-2) / 2, f \in$ $L^{m}(\Omega)$ with $1 \leq m \leq 1+(1 / p)$. Then, there is a weak solution $u \in W_{0}^{1.2}(\Omega) \cap L^{p+1}(\Omega)$ to problem $(1)$.

Proof. Set $f_{n}(x)=f(x) / 1+(1 / n)|f(x)|$ obviously, $f_{n}(x) \longrightarrow$ $f(x)$ in $L^{1}(\Omega)$ as $n \longrightarrow \infty$. Let $\phi=T_{k}\left(u_{n}\right)$ as a test function in (19), using the Hölder inequality and the Hardy inequality, we get 


$$
\begin{aligned}
\int_{\Omega} M(x) \nabla u_{n} \cdot \nabla T_{k}\left(u_{n}\right)+\int_{\Omega}\left|u_{n}\right|^{p-1} u_{n} T_{k}\left(u_{n}\right) \\
=\int_{\Omega} \frac{u_{n}}{1+(1 / n)\left|u_{n}\right|} \frac{E(x)}{1+(1 / n)|E(x)|} \\
\quad \cdot \nabla T_{k}\left(u_{n}\right)+\int_{\Omega} f_{n}(x) T_{k}\left(u_{n}\right) \\
\leq A \int_{\Omega} \frac{u_{n}}{|x|}\left|\nabla T_{k}\left(u_{n}\right)\right|+k \int_{\Omega}|f(x)| \\
=A \int_{\Omega} \frac{T_{k}\left(u_{n}\right)}{|x|}\left|\nabla T_{k}\left(u_{n}\right)\right|+k \int_{\Omega}|f(x)| \\
\leq \frac{A}{H} \int_{\Omega}\left|\nabla T_{k}\left(u_{n}\right)\right|^{2}+k \int_{\Omega}|f(x)| .
\end{aligned}
$$

Moreover, by (2) and a direct calculation, we get

$$
\begin{aligned}
\alpha \int_{\Omega}\left|\nabla T_{k}\left(u_{n}\right)\right|^{2}+\int_{\Omega} T_{k}^{p+1}\left(u_{n}\right) \leq & \int_{\Omega} M(x) \nabla T_{k}\left(u_{n}\right) \cdot \nabla T_{k}\left(u_{n}\right) \\
& +\int_{\Omega}\left|u_{n}\right|^{p} T_{k}\left(u_{n}\right) .
\end{aligned}
$$

Since $T_{k}\left(u_{n}\right) \in W_{0}^{1,2}(\Omega)$, by Lemma 10 , we get $m=1$ $+(1 / p)$. Together (35) with (36), it follows that

$$
\left(\alpha-\frac{A}{H}\right) \int_{\Omega}\left|\nabla T_{k}\left(u_{n}\right)\right|^{2}+\int_{\Omega} T_{k}^{p+1}\left(u_{n}\right) \leq C(\alpha, N, f, k) .
$$

We deduce that $T_{k}\left(u_{n}\right)$ is uniformly bounded in $W_{0}^{1,2}$ $(\Omega) \cap L^{p+1}(\Omega)$. Then, we pass to the limit in the approximation problem (19); up to a subsequence, there is a function $u \in W_{0}^{1,2}(\Omega) \cap L^{p+1}(\Omega)$.

Now, we want to prove that $\left|u_{n}\right|^{p} \longrightarrow|u|^{p}$. Let $\psi_{i}(\sigma)$ be defined by

$$
\psi_{i}(\sigma)= \begin{cases}1, & \sigma \geq t \\ 0, & |\sigma|<t \\ -1, & \sigma \leq-t\end{cases}
$$

Selecting $\phi=\psi_{i}\left(u_{n}\right)$ as a test function in (19), we have

$$
\int_{\Omega}\left|u_{n}\right|^{p} \psi_{i}\left(u_{n}\right) \leq \int_{\Omega} f \psi_{i}\left(u_{n}\right),
$$

which implies that

$$
\int_{\left\{u_{n}>t\right\} \cap \Omega}\left|u_{n}\right|^{p} \leq \int_{\left\{u_{n}>t\right\} \cap \Omega} f .
$$

Let $E \subset \Omega$ is measurable. For any $t>0$, we get

$$
\int_{E}\left|u_{n}\right|^{p} \leq t^{p}|E|+\int_{E \cap\left\{u_{n}>t\right\}}\left|u_{n}\right|^{p} \leq t^{p}|E|+\int_{\left\{u_{n}>t\right\}}|f| .
$$

The above fact and $f \in L^{1}(\Omega)$ allow us to say that, for any given $\varepsilon>0$, there is $t_{\varepsilon}$ satisfies

$$
\int_{\left\{u_{n}>t_{\varepsilon}\right\}}|f| \leq \varepsilon
$$

Thus,

$$
\int_{E}\left|u_{n}\right|^{p} \leq t_{\varepsilon}^{p}|E|+\varepsilon
$$

Therefore,

$$
\lim _{|E| \rightarrow 0} \int_{E}\left|u_{n}\right|^{p} \leq \varepsilon
$$

Thus, we prove that $\lim _{|E| \rightarrow 0} \int_{E}\left|u_{n}\right|^{p}=0$. Vitali theorem implies that $\left|u_{n}\right|^{p} \longrightarrow|u|^{p}$ in $L^{1}(\Omega)$. In other words,

$$
\lim _{n \rightarrow \infty} \int_{\Omega}\left|u_{n}\right|^{p}=\int_{\Omega}|u|^{p}
$$

Finally, let us show the second part of Theorem 1, that is, the existence of solution to problem (1) in the case where $f$ $\in L^{m}(\Omega)$ with $m>p+1 / p$.

Lemma 12. Assume that $f \in L^{m}(\Omega)$ with $m>p+1 / p$, and

$$
A \leq \frac{\alpha(N-2)}{p(m-1)+1}
$$

Then, there is a weak solution $u$ to problem (1) that such that

$$
\|u\|_{L^{m_{p}(\Omega)}} \leq C\|f\|_{L^{m}(\Omega)}
$$

where

$$
m_{p}=\frac{(p(m-1)+1) N}{N-2} .
$$

Proof. Define $\beta=p(m-1)>1$, it satisfies $p+\beta=\beta m^{\prime}$. Applying $\phi=\left|u_{n}\right|^{\beta-1} u_{n}$ as a test function in (19), by (3), we get

$$
\begin{aligned}
\int_{\Omega} M(x) \nabla u_{n} \cdot \nabla\left|u_{n}\right|^{\beta-1} u_{n}+\int_{\Omega}\left|u_{n}\right|^{p+\beta} \\
=\int_{\Omega} \frac{u_{n}}{1+(1 / n)\left|u_{n}\right|} \frac{E(x)}{1+(1 / n)|E(x)|} \\
\quad \cdot \nabla\left|u_{n}\right|^{\beta-1} u_{n}+\int_{\Omega} f_{n}(x)\left|u_{n}\right|^{\beta-1} u_{n} \\
\leq\left.\left. A \int_{\Omega} \frac{u_{n}}{|x|}|\nabla| u_{n}\right|^{\beta-1} u_{n}\left|+\int_{\Omega} f_{n}(x)\right| u_{n}\right|^{\beta-1} u_{n} \\
=A \beta \int_{\Omega} \frac{\left|u_{n}\right|^{\beta}}{|x|}\left|\nabla u_{n}\right|+\int_{\Omega} f_{n}(x)\left|u_{n}\right|^{\beta-1} u_{n} .
\end{aligned}
$$


By (2), (49) can become

$$
\begin{aligned}
& \alpha \beta \int_{\Omega}\left|\nabla u_{n}\right|^{2}\left|u_{n}\right|^{\beta-1}+\int_{\Omega}\left|u_{n}\right|^{p+\beta} \\
& \quad \leq A \beta \int_{\Omega} \frac{\left|u_{n}\right|^{\beta}}{|x|}\left|\nabla u_{n}\right|+\int_{\Omega} f_{n}(x)\left|u_{n}\right|^{\beta-1} u_{n} .
\end{aligned}
$$

Since

$$
\alpha \beta \int_{\Omega}\left|\nabla u_{n}\right|^{2}\left|u_{n}\right|^{\beta-1}=\left.\left.\frac{4 \alpha \beta}{(\beta+1)^{2}} \int_{\Omega}|\nabla| u_{n}\right|^{\beta+1 / 2}\right|^{2} .
$$

Applying the Hölder inequality and the Hardy inequality for the first term on the right-hand side of (50), it can be rewritten as

$$
\begin{aligned}
& \left.\left.\frac{4 \alpha \beta}{(\beta+1)^{2}} \int_{\Omega}|\nabla| u_{n}\right|^{\beta+1 / 2}\right|^{2}+\int_{\Omega}\left|u_{n}\right|^{p+\beta} \\
& \leq\left.\left.\frac{2 A \beta}{(\beta+1) H} \int_{\Omega}|\nabla| u_{n}\right|^{\beta+1 / 2}\right|^{2}+\int_{\Omega} f_{n}(x)\left|u_{n}\right|^{\beta-1} u_{n} .
\end{aligned}
$$

We conclude that

$$
\begin{gathered}
\left.\left.\left(\frac{4 \alpha \beta}{(\beta+1)^{2}}-\frac{2 A \beta}{(\beta+1) H}\right) \int_{\Omega}|\nabla| u_{n}\right|^{\beta+1 / 2}\right|^{2}+\int_{\Omega}\left|u_{n}\right|^{p+\beta} \\
\leq \int_{\Omega} f_{n}(x)\left|u_{n}\right|^{\beta-1} u_{n} \leq\|f\|_{L^{m}(\Omega)}\left(\int_{\Omega}\left|u_{n}\right|^{\beta m^{\prime}}\right)^{1 / m^{\prime}} .
\end{gathered}
$$

With this choice of $\beta$, by Lemma 10 , we arrive at

$$
\left.\left.\int_{\Omega}|\nabla| u_{n}\right|^{\beta+1 / 2}\right|^{2} \leq C\|f\|_{L^{m}(\Omega)} .
$$

According to the Sobolev embedding theorem, we obtain

$$
\left(\int_{\Omega}\left|u_{n}\right|^{(\beta+1) 2^{*} / 2}\right)^{1 / 2^{*}} \leq C\|f\|_{L^{m}(\Omega)} .
$$

Moreover,

$$
\frac{(\beta+1) 2^{*}}{2}=\frac{N(p(m-1)+1)}{N-2}=m_{p}
$$

where $2^{*}=2 N / N-2$.

As a consequence, there exists a function $|u| \epsilon$ $L^{(\beta+1) 2^{*} / 2}(\Omega)$.

Similarly, we can prove $\left|u_{n}\right|^{p} \longrightarrow|u|^{p}$. Then, we have proved the existence result.

Proof of Theorem 1. We can combine Lemmas 11-12 to get Theorem 1.

\section{Data Availability}

No data were used to support this study.

\section{Conflicts of Interest}

All authors declare that they have no competing interests.

\section{Authors' Contributions}

All authors contributed equally to this work.

\section{Acknowledgments}

This research was partially supported by the Program for Yong Talent of State Ethnic Affairs Commission of China (No. XBMU-2019-AB-34), Fundamental Research Funds for the Central Universities (No.31920200036), Innovation Team Project of Northwest Minzu University (No.1110130131), General Topics of National Education and Scientific Research (No. ZXYB18019), and First-rate Discipline of Northwest Minzu University.

\section{References}

[1] L. Boccardo, "Some developments on Dirichlet problems with discontinuous coefficients," Bollettino Dell Unione Matematica Italiana, vol. 2, no. 2, pp. 285-297, 2009.

[2] L. Boccardo, "Dirichlet problems with singular convection terms and applications," Journal of Differential Equations, vol. 258, no. 7, pp. 2290-2314, 2015.

[3] L. Boccardo and L. Orsina, "Very singular solutions for linear Dirichlet problems with singular convection terms," Nonlinear Analysis, vol. 194, article 111437, p. 18, 2020.

[4] D. Arcoya, L. Boccardo, and L. Orsina, "Regularizing effect of the interplay between coefficients in some nonlinear Dirichlet problems with distributional data," Annali di Matematica Pura ed Applicata, vol. 199, no. 5, pp. 1909-1921, 2020.

[5] L. Boccardo, "Stampacchia-Calderon-Zygmund theory for linear elliptic equations with discontinuous coefficients and singular drift," ESAIM Control Optimisation and Calculus of Variations, vol. 25, article 47, p. 18, 2019.

[6] H. Brezis and J. Vazquez, "Blow-up solutions of some nonlinear elliptic problems," Revista Matemática Complutense, vol. 10, no. 2, pp. 443-470, 1997.

[7] G. Croce, "The regularizing effects of some lower order terms in an elliptic equation with degenerate coercivity," Ricerche di Matematica, vol. 27, pp. 299-314, 2010.

[8] C. De Coster and L. Jeanjean, "Multiplicity results in the noncoercive case for an elliptic problem with critical growth in the gradient," Journal of Differential Equations, vol. 262, no. 10, pp. 5231-5270, 2017.

[9] D. Giachetti and M. M. Porzio, "Elliptic equations with degenerate Coercivity: gradient regularity," Acta Mathematica Sinica, English Series, vol. 19, no. 2, pp. 349-370, 2003.

[10] L. Gasiński and N. S. Papageorgiou, "Positive solutions for nonlinear elliptic problems with dependence on the gradient," Journal of Differential Equations, vol. 263, no. 2, pp. 14511476, 2017. 
[11] L. Gasiński, L. Klimczak, and N. S. Papageorgiou, "Nonlinear noncoercive Neumann problems," Communications on Pure and Applied Analysis, vol. 15, no. 4, pp. 1107-1123, 2016.

[12] S. Huang, T. Su, X. Du, and X. Zhang, "Entropy solutions to noncoercive nonlinear elliptic equations with measure data," Electronic Journal of Differential Equations, vol. 2019, no. 97, pp. 1-22, 2019.

[13] S. Huang and Q. Tian, "Harnack-type inequality for fractional elliptic equations with critical exponent," Mathematical Methods in the Applied Sciences, vol. 43, no. 8, pp. 53805397, 2020.

[14] S. Huang, Q. Tian, J. Wang, and J. Mu, "Stability for noncoercive elliptic equations," Electronic Journal of Differential Equations, vol. 2016, no. 242, pp. 1-11, 2016.

[15] M. Ri, S. Huang, and C. Huang, "Non-existence of solutions to some degenerate coercivity elliptic equations involving measures data," Electronic Research Archive, vol. 28, no. 1, pp. 165-182, 2020.

[16] J. Xiawu, S. Huang, Y. Mi, and M. Ri, "Existence of $W_{0}^{1,1}(\Omega)$ solutions to non-coercivity quasilinear elliptic problem," Journal of Function Spaces, vol. 2020, Article ID 5017818, 6 pages, 2020.

[17] L. Adimurthi, G. C. Boccardo, and L. Orsina, "The regularizing effect of lower order terms in elliptic problems involving Hardy potential," Advanced Nonlinear Studies, vol. 17, no. 2, pp. 311-317, 2017.

[18] D. Arcoya, L. Boccardo, and T. Leonori, " $W_{0}^{1,1}$-solutions for elliptic problems having gradient quadratic lower order terms," Nonlinear Differential Equations and Applications, vol. 20, no. 6, pp. 1741-1757, 2013.

[19] D. Arcoya and L. Boccardo, "Regularizing effect of the interplay between coefficients in some elliptic equations," Journal of Functional Analysis, vol. 268, no. 5, pp. 1153-1166, 2015.

[20] D. Arcoya and L. Boccardo, "Regularizing effect of $L^{q}$ interplay between coefficients in some elliptic equations," Journal de Mathématiques Pures et Appliquées, vol. 111, no. 1, pp. 106$125,2018$.

[21] D. Arcoya, L. Boccardo, T. Leonori, and A. Porretta, "Some elliptic problems with singular natural growth lower order terms," Journal of Differential Equations, vol. 249, no. 11, pp. 2771-2795, 2010.

[22] L. Boccardo, T. Gallouet, and J. Vazquez, "Nonlinear elliptic equations in $\mathbb{R}^{N}$ without growth restrictions on the data," Journal of Differential Equations, vol. 105, no. 2, pp. 334$363,1993$.

[23] L. Boccardo and L. Orsina, "Regularizing effect of the lower order terms in some elliptic problems: old and new," Rendiconti Lincei - Matematica e Applicazioni, vol. 29, no. 2, pp. 387-399, 2018.

[24] G. Cirmi, "Regularity of the solutions to nonlinear elliptic equations with a lower-order term," Nonlinear Analysis, vol. 25 , no. 6 , pp. 569-580, 1995.

[25] G. Chen, "Nonlinear elliptic equation with lower order term and degenerate coercivity," Mathematical Notes, vol. 93, no. 1-2, pp. 224-237, 2013.

[26] Y. Mi, S. Huang, and C. Huang, "Combined effects of the Hardy potential and lower order terms in fractional Laplacian equations," Boundary Value Problems, vol. 2018, Article ID 61, 12 pages, 2018.
[27] L. Boccardo, S. Buccheri, and G. R. Cirmi, "Two linear noncoercive Dirichlet problems in duality," Milan Journal of Mathematics, vol. 86, no. 1, pp. 97-104, 2018.

[28] J. Garcia Azorero and I. Peral Alonso, "Hardy inequalities and some critical elliptic and parabolic problems," Journal of Differential Equations, vol. 144, no. 2, pp. 441-476, 1998.

[29] J. L. Vázquez and E. Zuazua, "The Hardy inequality and the asymptotic behaviour of the heat equation with an inversesquare potential," Journal of Functional Analysis, vol. 173, no. 1, pp. 103-153, 2000. 